\title{
A Note on Compatibility Relation
}

\author{
${ }^{1}$ T.O William-west (research scholar) and ${ }^{2} Y$. S Daniel \\ ${ }^{I}$ Department of Mathematics, Ahmadu Bello University, Zaria, Nigeria. \\ ${ }^{2}$ Department of Mathematics, Kaduna State University, Kaduna, Nigeria.
}

\begin{abstract}
A lot of literatures characterize an extension of an order on a set (say, $X)$ to a weak order on the set of all of its non- empty subsets (say, F). This extension makes use of the information contained in the primitive order on $X$. This paper considers the case where the subsets of $X$ are formed by a compatibility relation defined on X. As its main contribution, the paper attempts to show that an ordering can be defined on the collection of compatible subsets of a finite set endowed with a compatibility relation. In particular, a necessary and sufficient condition is formulated that would guarantee when an ordering so defined turns out to be partial ordering.
\end{abstract}

Keywords: Compatibility relation, covering, ordering

\section{Introduction}

Essentially, a compatibility relation defined on a finite set decomposes the set into its possibly nondisjoint subsets, henceforth called compatibility sets. It may often happen that none of these compatibility sets in this decomposition is a subset of another. Thus the idea of ordering a collection of compatibility sets in terms of subsethood relation is out of the question. On the other hand, if a compatibility relation is defined on an ordered set $\mathrm{X}$, it makes sense to ask whether the ordering of $\mathrm{X}$ can be extended to the collection of its compatibility sets. A lot of literatures characterize an extension of an order on a set (say, $\mathrm{X}$ ) to a weak order on the set of all of its non- empty subsets (say, F). This extension makes use of the information contained in the primitive order on $\mathrm{X}$ (see [2], for some details).Usually, a lot of problems in individual and collective decision making involve the comparism of sets of objects (see [3], for some details). For many practical problems, only information about preferences among single objects is available. Consequently, the idea of ordering (or even how to derive a ranking over) the set of all non- empty subsets of $\mathrm{X}$ in a way that preserves the primitive ordering relation on $\mathrm{X}$ is a fundamental question.

This question, for the case of ranking of sets has been considered in some literatures (see [3], and the references there for some details). Also, in [4], the question has been carried out in the tradition of the literature on extending an order on a set to its power set with the objective to axiomatically characterize families of ordinal preferences over subsets.

This paper considers the case where the subsets of $\mathrm{X}$ are formed by a compatibility relation defined on $\mathrm{X}$, and focus on some criteria and method(s) for ordering compatibility sets of $\mathrm{X}$. This situation can be observed in many different contexts, for example, we may consider the problem of what to take on a backpacking trip on the mountains: a "bottle of water" can be more essential (thus orders the rest objects) than a "bottle of orange juice" or than a "sandwich" or than a "doughnut", and a "bottle of orange juice" could be preferred (e.g., for dietary reasons) to "sandwich" or to "doughnut", and "sandwich" could be preferred (for some other reasons) to "doughnut". But if some compatibility relation is defined on these objects (for food classification or necessity reasons) which pairs drinks and snacks in compatibility sets A and B respectively, it is observed that the decision making becomes a comparism (or some kind of ordering) of these sets of objects, if the primitive ordering relation on these objects is preserved. This characterization in reality is more complex, though. The example above is constructively obtained from [3], only that we consider a case where compatibility relation is defined among these objects.

In this paper, we attempt to show that to what extent the ordering defined on a given ordered finite set (endowed with a compatibility relation) is extendible to the collection of its compatibility sets. In particular, we give a necessary and sufficient condition that would guarantee when such an extension turns out to be partial, linear or well ordering.

We note here that unlike [2], [3] and [4], elements of $\mathrm{X}$ are interpreted as possible compatible options rather than exclusive alternatives.

\section{Notation and definitions}

Let $S$ be a set with $n$ elements, usually denoted $n$ - set. A family $\left\{A_{1}, A_{2}, A_{3}, \ldots, A_{n}\right\}$ of non- empty subsets of $\mathrm{S}$ is called covering of $\mathrm{S}$ if $\mathrm{S}=\mathrm{U}_{k=1}^{\mathrm{n}} A_{k}$. For example, the set $\mathrm{S}_{1}=\{\mathrm{a}\}$ has only a single cover, namely; $\{\{a\}\}$. However, there are five covers of the set $S_{2}=\{a, b\}$, namely; $\{\{a\},\{b\}\},\{\{a, b\}\},\{\{a\},\{a$, $b\}\},\{\{b\},\{a, b\}\}$ and $\{\{a\},\{b\},\{a, b\}\}$. Note that $A_{k}$ 's are not necessarily disjoint, and hence it may not define a partition. 
A relation on a set $\mathrm{S}$ which is reflexive and symmetric is called a compatibility relation, sometimes denoted $\approx$. Also, let $R$ be a compatibility relation on a finite set $S$, then $x, y \in S$ are called compatible if $x$ Ry (see [1], for details).

Note that compatibility relation not being necessary transitive may not define a partition. However, it does define a covering (see [1], for details).

For example, consider the set $S=\left\{x_{1}, x_{2}, x_{3}, x_{4}, x_{5}\right\}=\{\{1,2\},\{2,3,4\},\{3,4,5\},\{2,4\},\{4,5\}\}$ and let $R$ be given by: $R=\left\{\left(x_{i}, x_{j}\right) \mid x_{i}, x_{j} \in S \wedge x_{i} R x_{j}\right.$ if $x_{i}$ and $x_{j}$ contain some common element $\}$. Clearly, $x_{1} R x_{2} \wedge x_{2} R x_{3} \nRightarrow$ $\mathrm{x}_{1} \mathrm{Rx}_{3}$.

From the diagram below, it is easy to observe that the elements in each of the sets $\left\{\mathrm{x}_{1}, \mathrm{x}_{2}, \mathrm{x}_{4}\right\},\left\{\mathrm{x}_{2}, \mathrm{x}_{3}, \mathrm{x}_{4}\right\},\left\{\mathrm{x}_{2}\right.$, $\left.\mathrm{x}_{4}, \mathrm{x}_{5}\right\},\left\{\mathrm{x}_{3}, \mathrm{x}_{4}, \mathrm{x}_{5}\right\}$ and $\left\{\mathrm{x}_{2}, \mathrm{x}_{3}, \mathrm{x}_{4}, \mathrm{x}_{5}\right\}$ are mutually compatible and the sets are not mutually disjoint.

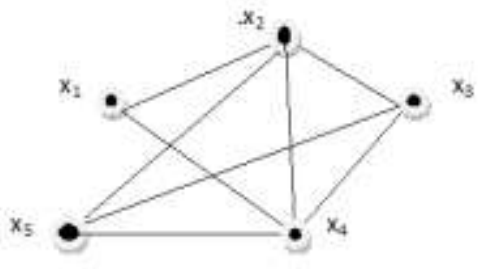

Fig. 1: Graph of R.

\section{Context in which ordering compatibility sets is important}

Let $\mathrm{X}$ be a finite set of ordered objects (goods) upon which a compatibility relation is defined. Suppose now that an agent's preference for goods in $\mathrm{X}$ defines an ordering on $\mathrm{X}$ and a compatibility relation $\mathrm{R}$ (for similar reasons discussed in section 1) which decomposes $X$ into sets $A_{1}=\left\{x_{1}, x_{2}, x_{3}\right\}$ and $A_{2}=\left\{x_{1}, x_{2}\right\}$ at some time t. What might be appreciated from $A_{1}$ is that; at some time $t+1$, it provides more flexibility than $A_{2}$. Suppose, again, that the agent will make a final choice at some later time, the set $A_{1}$ may be viewed as the set of options it will have at some time $\mathrm{t}+\mathrm{k}$ (for $\mathrm{k} \geq 1)$.

Following the above, in the next section, we attempt to characterize an extension of the primitive ordering relation on $\mathrm{X}$ to the collection of compatibility sets, such that the choice of the agent is reasonably captured.

\section{Main results}

Basically, since our interest lies in extending the ordering relation on $\mathrm{X}$ to the collection of its compatibility sets, we purpose to introduce at this juncture a new definition.

Definition 3.1

Let $\leqslant$ be an ordering relation on $X$. Let $\approx$ be a compatibility relation defined on $X$.We say that $\leqslant$ is extendible to $\subseteq$ on the collection of compatibility sets of $X$ if, for $x, y \in X, x \leqslant y$ implies that $C(x) \subseteq C(y)$, where $\mathrm{C}(\mathrm{x})$ and $\mathrm{C}(\mathrm{y})$ are compatibility sets containing $\mathrm{x}$ and $\mathrm{y}$ respectively, and $ᄃ$ is an extension of $\leqslant$.

Note that if $x \leqslant y$, then it will be contextually understood that $\{x\} \subseteq\{y\}$. Similarly, if $x \leqslant y \leqslant z$, then $\{x, y\} \subseteq$ $\{\mathrm{y}, \mathrm{z}\}$, for all $\mathrm{x}, \mathrm{y}, \mathrm{z} \in \mathrm{X}$. By so doing, note that no possible effects of complementarities between $\mathrm{x}$ and $\mathrm{z}$ (or because of incompatibility between $x$ and $y$ ) is assumed to reverse the ordering relation of sets $\{x, y\}$ and $\{y, z\}$, since each element in the sets are compatible and are (primitively) ordered in $\mathrm{X}$. However, for the purpose of ordering compatibility sets of $X$ such as; $A_{1}=\{w, y, z\}$ and $A_{2}=\{w, x, y, z\}$ or $A_{3}=\{w, z\}$ and $A_{4}=\{x, y\}$, whenever $\mathrm{w} \leqslant \mathrm{x} \leqslant \mathrm{y} \leqslant \mathrm{z}$. This suggests for introducing a new definition on $\mathrm{F}$ - the family of compatibility sets of $\mathrm{X}$. Nonetheless, it could be argued that $\mathrm{A}_{2}$ may be appreciated than $\mathrm{A}_{1}$, since it provides more flexibility or options. For the sake of $\mathrm{A}_{3}$ and $\mathrm{A}_{4}$, we give the following definition.

\section{Definition 3.2}

1. Let $\mathrm{X}$ be a finite set and $\leqslant$ an ordering relation on $\mathrm{X}$. We say the order of an element $\mathrm{x} \in \mathrm{X}$ denoted $\mathrm{o}(\mathrm{x})$ is $\mathbf{r}$ if there exist $\mathrm{g}: \mathrm{X} \rightarrow \boldsymbol{\beta} \subset \boldsymbol{R}$ defined by $\mathrm{g}(\mathrm{x})=\mathbf{r}=\mathrm{o}(\mathrm{x})$, where $\mathbf{r}$ is a numerical representation of the position of $\mathrm{x}$ in $\mathrm{X}$ with respect to $\leqslant$.

2. Let $\mathrm{g}: \mathrm{X} \rightarrow \boldsymbol{\beta} \subset \boldsymbol{R}$ and $\mu: \mathrm{F} \rightarrow \boldsymbol{\alpha}$ be maps defined by $\mathrm{g}(\mathrm{x})=\mathrm{o}(\mathrm{x})$ and $\mu(\mathrm{C})=\mathbf{a}=\sum_{\mathrm{i}} g\left(x_{i}\right)$ respectively, $\mathrm{x}_{\mathrm{i}}$ $\in C \in F$, for all $i$ and $\boldsymbol{\alpha}$ an ordered set of numbers containing a. We say $C_{1} \subseteq C_{2} \Leftrightarrow \mu\left(C_{1}\right) \leq \mu\left(C_{2}\right)$ and $C_{1} \sqsubset$ $\mathrm{C}_{2} \Leftrightarrow \mu\left(\mathrm{C}_{1}\right)<\mu\left(\mathrm{C}_{2}\right)$, for $\mathrm{C}_{1}, \mathrm{C}_{2} \in \mathrm{F}$.

\section{Proposition 3.1}

Let $\mathrm{X}$ be a finite set endowed with a compatibility relation $\mathrm{R}$. Let $\leqslant$ be a partial ordering relation on $\mathrm{X}$. Then $\leqslant$ is extendible to $\subseteq^{*}$ on the collection $\mathrm{F}$ of compatible sets of $\mathrm{X}$. 
Proof: We wish to show that a partial ordering relation could be defined on $\mathrm{F}$ whenever $\leqslant$ is a partial order on $X$. To see this, define a map $\varphi: X \rightarrow F$ by $\varphi(x)=\{\mathrm{y} \in \mathrm{X}: \mathrm{y} \leqslant \mathrm{X}\}$. By this map, $X$ is isomorphic to the range of $\varphi$ ordered by $\subseteq$. Our claim follows from the fact that: if $\mathrm{x} \leqslant \mathrm{y}$, then $\mathrm{z} \leqslant \mathrm{x}$ implies $\mathrm{z} \leqslant \mathrm{y}$ by transitivity of $\leqslant$, and hence $\varphi(\mathrm{x}) \subseteq \varphi(\mathrm{y})$. Since $\mathrm{x} \in \varphi(\mathrm{x})$ (by reflexivity of $\preccurlyeq), \varphi(\mathrm{x}) \subseteq \varphi(\mathrm{y})$ implies $\mathrm{x} \leqslant \mathrm{y}$. Thus, $\mathrm{x} \leqslant \mathrm{y} \Leftrightarrow \varphi(\mathrm{x})$ $\sqsubseteq \varphi(\mathrm{y})$.

That $\varphi$ is $1-1$ then follows by antisymmetry of $\leqslant$. Hence by the nature of $\varphi$ (order embedding), $\mathrm{F}$ must be partially ordered.

We note that $\varphi(\mathrm{x})=\mathrm{C} \in \mathrm{F}$, for compatibility set $\mathrm{C}$ and $\subseteq^{*} \equiv$ ㄷ․

\section{Proposition 3.2}

Let $X$ be a finite set upon which a compatibility relation is defined and $\leqslant$ a partial ordering relation on $X$. Let $\mathrm{g}: \mathrm{X} \rightarrow \boldsymbol{\beta} \subset \boldsymbol{R}$ be a map defined by $\mathrm{x} \leqslant \mathrm{y} \Leftrightarrow \mathrm{g}(\mathrm{x}) \leqslant \mathrm{g}(\mathrm{y})$, for $\mathrm{x}, \mathrm{y} \in \mathrm{X}$, where $\mathrm{g}(\mathrm{x})=\mathrm{o}(\mathrm{x})$. Then the collection $\mathrm{F}$ of compatibility sets of $\mathrm{X}$ is partially ordered if and only there exist a bijective mapping $\mu: \mathrm{F} \rightarrow \boldsymbol{\alpha}$ defined by $\mu(\mathrm{C})=\mathbf{a}=\sum_{\mathrm{i}} g\left(x_{i}\right), \mathrm{x}_{\mathrm{i}} \in \mathrm{C} \in \mathrm{F}$, for all i and $\boldsymbol{\alpha}$ a partially ordered set of numbers (which extends $\beta$ ) containing a.

Proof: Suppose F is partially ordered, by 2 of definition 3.2, there exists a mapping $\mu$. We wish to show that $\mu$ is bijective.

To see this, let $C_{1}$, and $C_{2}$ be distinct members of $F$. Then there exist an element $x \in C_{1}$ with $x \notin C_{2}$ (or $x \in C_{2}$ with $\left.\mathrm{x} \notin \mathrm{C}_{1}\right)$. Thus, $\sum_{\mathrm{i}} g\left(x_{i}\right) \in \mu\left(\mathrm{C}_{1}\right)$, for $\mathrm{x}_{\mathrm{i}} \in \mathrm{C}_{1}$ and since $\mathrm{g}$ is $1-1, \Sigma_{\mathrm{i}} g\left(x_{i}\right) \notin \mu\left(\mathrm{C}_{2}\right)$, for $\mathrm{x}_{\mathrm{i}} \in \mathrm{C}_{1}$ (or $\Sigma_{i} g\left(x_{i}\right) \in \mu\left(C_{2}\right)$, for $x_{i} \in C_{2}$ and $\sum_{i} g\left(x_{i}\right) \notin \mu\left(C_{1}\right)$, for $\left.x_{i} \in C_{2}\right)$. Hence $\mu\left(C_{1}\right) \neq \mu\left(C_{2}\right)$. Therefore, $\mu$ is $1-1$. Also, $\mathbf{a} \in \boldsymbol{\alpha}$, since $\mathrm{g}$ is onto, $\mu^{-1}(\mathbf{a})=\left\{\mathrm{x} \mid \mathrm{x} \in \mathrm{X}, \Sigma_{\mathrm{i}} g\left(x_{i}\right) \in \alpha\right\}$ is not empty. But a is the image of $\mu^{-1}(\mathbf{a})$, i.e., $\mu\left(\mu^{-1}(\mathbf{a})\right)=\mathbf{a}$. Hence $\mu$ is onto.

The converse of the result follows from the fact that $\mu$ is bijective and $g$ is an order preserving mapping.

\section{Example 3.1}

Let $X=\left\{x_{1}, x_{2}, x_{3}, x_{4}, x_{5}\right\}=\{\{2,3,4\},\{4,5,5\},\{3,5,7\},\{1,2,6,6\},\{4,6,8\}\}$, where $x_{1}=\{2,3,4\}$, etc., respectively. Define a compatibility relation $R$ on $X$ by: $R=\left\{\left(x_{i}, x_{j}\right) \mid x_{i}, x_{j} \in S \wedge x_{i} R x_{j}\right.$ if $x_{i}$ and $x_{j}$ contain some common element\}.

Clearly, $\{1,2,6,6\} R\{2,3,4\}$ and $\{2,3,4\} R\{4,5,5\} \Rightarrow\{1,2,6,6\} R\{4,5,5\}$.

We may define an ordering relation on $\mathrm{X}$ as follows:

Let $\leqslant$ be a partial ordering relation on $X$ if and only if there exists a lexicographic ordering relation $\leqslant^{\text {lex }}$ such that $\mathrm{x} \leqslant^{l e x} \mathrm{y}$, for $\mathrm{x}, \mathrm{y} \in \mathrm{X}$. Note that the elements of $\mathrm{X}$ could be multisets (a multiset is a collection in which objects are allowed to repeat).

Now, define a mapping $\mathrm{g}: \mathrm{X} \rightarrow \boldsymbol{\beta} \subset \boldsymbol{R}$, by $\mathrm{g}(\mathrm{x})=\mathbf{r} \in \boldsymbol{\beta}$, such that $\mathrm{g}\left(\mathrm{x}_{1}\right)=0.01, \mathrm{~g}\left(\mathrm{x}_{2}\right)=0.011, \mathrm{~g}\left(\mathrm{x}_{3}\right)=0.0111$, $\mathrm{g}\left(\mathrm{x}_{4}\right)=0.01111$ and $\mathrm{g}\left(\mathrm{x}_{5}\right)=0.011111$, where $\boldsymbol{\beta}=\{0.01,0.011,0.0111,0.01111,0.011111\}$.

Let $F$ be the collection of compatibility subsets of $X$. Then $F=\left\{\left\{x_{1}, x_{2}, x_{5}\right\},\left\{x_{2}, x_{3}\right\},\left\{x_{4}, x_{5}\right\},\left\{x_{1}, x_{3}\right\},\left\{x_{1}\right.\right.$, $\left.\left.\mathrm{x}_{4}\right\}\right\}=\left\{\mathrm{C}_{1}, \mathrm{C}_{2}, \mathrm{C}_{3}, \mathrm{C}_{4}, \mathrm{C}_{5}\right\}$, where $\mathrm{C}_{1}=\left\{\mathrm{x}_{1}, \mathrm{x}_{2}, \mathrm{x}_{5}\right\}$, etc., respectively. Define $\mu: \mathrm{F} \rightarrow \boldsymbol{\alpha}$ defined by $\mu(\mathrm{C})$ $=\Sigma_{i} g\left(x_{i}\right), x_{i} \in C \in F$, such that $\mu\left(\mathrm{C}_{1}\right)=0.032111, \mu\left(\mathrm{C}_{2}\right)=0.0221, \mu\left(\mathrm{C}_{3}\right)=0.022221, \mu\left(\mathrm{C}_{4}\right)=0.0211$ and $\mu\left(\mathrm{C}_{1}\right)$ $=0.02111$, where $\alpha=\{0.032111,0.0221,0.022221,0.0211,0.02111\}$.

\section{Concluding remarks}

In this paper we have introduce a useful concept which aims at extending the primitive ordering on a finite set. The paper has attempted to show to what extent the ordering defined on a given finite set (endowed with a compatibility relation), is extendible to the collection of its compatibility sets. Particularly, a necessary and sufficient condition that would guarantee when such an extension turns out to be partial ordering was given. We have shown that an ordering can be defined on the collection of compatibility sets. The direction of our future research is to formulate a necessary and sufficient condition that would guarantee when this ordering turns out to be wellordering.

\section{References}

[1] D. Singh, M.H. Dunari and J.N. Singh, A Note on Covering and Partition of a Finite Set, Math. Sci. Math. Edu. 5(2) (2010), 1 - 5.

[2] N. Baigent and Y. Xu, Extending an Order on a Set to its Power Set: An Axiomatic Characterization of Average Borda Rule, Journal of Economic Theory, 49(5) (2004), 43- 56.

[3] S. Moretti and A. Tsoukias, Ranking Sets of Possibly Interacting Objects Using Shapley Extensions, International Conference on Principles of Knowledge and Reasoning, 12 (2010) 199- 207.

[4] S. Barbera and P.K. Pattanaik, Extending an Order on a Set to its Power Set: Some Remarks on Kannai and Peleg's Approach, Journal of Economic Theory 49 (1989), 84- 92. 